\title{
Article \\ A Markerless Gene Deletion System in Streptococcus suis by Using the Copper-Inducible Vibrio parahaemolyticus YoeB Toxin as a Counterselectable Marker
}

\author{
Chengkun Zheng ${ }^{1,2, *,+}$ C , Man Wei ${ }^{1,2,+}$, Jun Qiu ${ }^{1,2}$ and Jinquan $\mathrm{Li}^{3}$ \\ 1 Joint International Research Laboratory of Agriculture and Agri-Product Safety, The Ministry of Education of \\ China, Yangzhou University, Yangzhou 225009, China; fhzxmwei@163.com (M.W.); qiujun527@163.com (J.Q.) \\ 2 Jiangsu Key Laboratory of Zoonosis, Yangzhou University, Yangzhou 225009, China \\ 3 College of Food Science and Technology, Huazhong Agricultural University, Wuhan 430070, China; \\ lijinquan@mail.hzau.edu.cn \\ * Correspondence: zhengchengkun@yzu.edu.cn \\ + These authors contributed equally to this work.
}

check for

updates

Citation: Zheng, C.; Wei, M.; Qiu, J.; Li, J. A Markerless Gene Deletion System in Streptococcus suis by Using the Copper-Inducible Vibrio

parahaemolyticus YoeB Toxin as a Counterselectable Marker. Microorganisms 2021, 9, 1095. https://doi.org/10.3390/ microorganisms 9051095

Academic Editor:

Muhammad Kamruzzaman

Received: 5 April 2021

Accepted: 18 May 2021

Published: 19 May 2021

Publisher's Note: MDPI stays neutral with regard to jurisdictional claims in published maps and institutional affiliations.

Copyright: (c) 2021 by the authors. Licensee MDPI, Basel, Switzerland. This article is an open access article distributed under the terms and conditions of the Creative Commons Attribution (CC BY) license (https:/ / creativecommons.org/licenses/by/ $4.0 /)$.

\begin{abstract}
Streptococcus suis is an important zoonotic pathogen causing severe infections in swine and humans. Induction of the Vibrio parahaemolyticus YoeB toxin in Escherichia coli resulted in cell death, leading to the speculation that $Y_{o e} B_{V p}$ can be a counterselectable marker. Herein, the counterselection potential of $Y_{o e} B_{V p}$ was assessed in $S$. suis. The yoe $B_{V p}$ gene was placed under the copper-induced promoter PcopA. The PcopA-yoe $B_{V p}$ construct was cloned into the $S$. suis-E. coli shuttle vector pSET2 and introduced into $S$. suis to assess the effect of $Y_{o e} B_{V p}$ expression on $S$. suis growth. Reverse transcription quantitative PCR showed that copper induced yoe $B_{V p}$ expression. Growth curve analyses and spot dilution assays showed that $\mathrm{YoeB}_{V p}$ expression inhibited S. suis growth both in liquid media and on agar plates, revealing that $\mathrm{YoeB}_{V p}$ has the potential to be a counterselectable marker for $S$. suis. A SCIY cassette comprising the spectinomycin-resistance gene and copper-induced yoe $B_{V p}$ was constructed. Using the SCIY cassette and peptide-induced competence, a novel two-step markerless gene deletion method was established for $S$. suis. Moreover, using the $\Delta p e r R$ mutant generated by this method, we demonstrated that PmtA, a ferrous iron and cobalt efflux pump in S. suis, was negatively regulated by the PerR regulator.
\end{abstract}

Keywords: Streptococcus suis; markerless gene deletion; toxin; YoeB; counterselectable marker

\section{Introduction}

Streptococcus suis is a Gram-positive, facultative anaerobe that threatens the swine industry and public health worldwide [1]. It is responsible for various swine diseases, including meningitis, septicemia, pneumonia, endocarditis, and arthritis [2]. Generally, S. suis is considered one of the most important bacterial pathogens that lead to significant economic losses to the swine industry [3]. Indeed, a recent survey revealed that its isolation rate was $16.9 \%$, ranking first among the bacterial pathogens isolated from Chinese pig farms from 2013 to 2017 [4]. More seriously, S. suis can be transmitted to humans by minor skin injuries or the gastrointestinal tract, causing meningitis, streptococcal toxic shock-like syndrome, and some other clinical symptoms [5]. In 1968, the first human case of S. suis infection was described in Denmark; since then, over 1600 human cases have been reported worldwide by the end of 2013, some of which were fatal [6]. Remarkably, two great outbreaks of human S. suis infections occurred in China in 1998 and 2005, resulting in 25 cases with 14 deaths and 215 cases with 39 deaths, respectively [7,8]. In recent years, S. suis still frequently caused sporadic human cases worldwide [9-14].

Over the past few decades, significant progress has been made toward understanding the physiology and pathogenesis of S. suis. A number of virulence-related factors have been described in $S$. suis $[15,16]$. Recently, in vivo transcriptomes and transposon 
mutant libraries have been applied to identify genes involved in the virulence traits of S. suis [17-19]. Usually, studies related to the physiology and pathogenesis of S. suis rely on gene deletion mutants. In S. suis, the most frequently used gene deletion system is the pSET4s thermosensitive suicide vector [20]. For gene deletion using pSET4s, a knockout vector is constructed and introduced into the wild-type (WT) S. suis strain by electroporation; subsequently, the mutant is selected after two steps of allelic exchange. As this system contains no counterselectable marker, the mutant must be picked out from many potential colonies. In addition, electrotransformation does not work well for certain S. suis isolates [21]. Except for allelic exchange using the pSET4s vector, a cloning-independent method employing peptide-induced competence has been established in S. suis [22]. This method allows high-throughput mutation; however, the mutant carries a spectinomycin resistance gene, limiting its vaccine potential. Only recently, Zhu et al. developed a markerless gene deletion method in S. suis Chz serotype with the utilization of the ComRS system and sucrose sensitivity [21].

Toxin-antitoxin (TA) systems are small genetic modules widely distributed in the plasmids or chromosomes of bacteria and archaea [23]. Typically, they are composed of a gene encoding a stable toxin and a gene encoding an unstable antitoxin [24,25]. Under stress conditions, toxins are released from the TA complex and target various cellular functions to inhibit cell growth, making them valuable for counterselection [26-30]. In a previous study, we identified a chromosomal type II toxin-antitoxin system, YefM-YoeB, in Vibrio parahaemolyticus; induction of $\mathrm{YoeB}_{V p}$ in Escherichia coli resulted in cell death [31]. This result has led us to speculate that $Y_{0 e} B_{V p}$ can be a counterselectable marker for $S$. suis.

In this study, the $Y_{0 e B_{V p}}$ toxin was tested for the counterselection potential in S. suis. Using YoeB $B_{V p}$ as a counterselectable marker, we successfully established a novel two-step markerless gene deletion method for $S$. suis. Finally, using the $\Delta p e r R$ mutant generated by this method, we demonstrated that $p m t A$, a gene encoding a ferrous iron and cobalt efflux pump in S. suis [32] was negatively regulated by the PerR regulator.

\section{Materials and Methods}

\subsection{Bacterial Strains, Plasmids, Primers, and Culture Conditions}

Bacterial strains and plasmids used in this study are listed in Table 1. All primers are listed in Table S1. Unless otherwise specified, S. suis strains were cultured at $37^{\circ} \mathrm{C}$ in Tryptic Soy Broth (TSB) or on Tryptic Soy Agar (TSA; Becton, Dickinson and Company, Suzhou, China) supplemented with 5\% (vol/vol) newborn bovine serum (Sijiqing, Hangzhou, China). E. coli strains were grown in Luria-Bertani (LB) broth or on LB agar. When required, spectinomycin was added to the medium at $50 \mu \mathrm{g} / \mathrm{mL}$ for E. coli and $100 \mu \mathrm{g} / \mathrm{mL}$ for S. suis.

Table 1. Bacterial strains and plasmids used in this study.

\begin{tabular}{|c|c|c|}
\hline Strain or Plasmid & Relevant Characteristics ${ }^{1}$ & Source or Reference \\
\hline \multicolumn{3}{|l|}{ Strains } \\
\hline SC19 & $\begin{array}{c}\text { Virulent } S \text {. suis strain isolated from the } \\
\text { brain of a dead pig }\end{array}$ & {$[33]$} \\
\hline SC19/pSET2-PcopA-yoeB $V p$ & $\begin{array}{l}\text { Strain SC19 expressing the } \\
\text { copper-inducible } \text { YoeB }_{V p} \text { toxin }\end{array}$ & This study \\
\hline SC19/pSET2-PcopA-yoeB ${ }_{S s}$ & $\begin{array}{l}\text { Strain SC19 expressing the } \\
\text { copper-inducible } \text { YoeB }_{S s} \text { toxin }\end{array}$ & This study \\
\hline SC19/pSET2 & Strain SC19 carrying the pSET2 vector & This study \\
\hline$\Delta p m t A$ & $p m t A$ deletion mutant of strain SC19 & This study \\
\hline$\Delta p e r R$ & perR deletion mutant of strain SC19 & This study \\
\hline$\Delta l y s R$ & lysR deletion mutant of strain SC19 & This study \\
\hline MC1061 & Cloning host for recombinant vector & AngYuBio, Shanghai, China \\
\hline
\end{tabular}


Table 1. Cont.

\begin{tabular}{|c|c|c|}
\hline Strain or Plasmid & Relevant Characteristics ${ }^{1}$ & Source or Reference \\
\hline \multicolumn{3}{|l|}{ Plasmids } \\
\hline pSET2 & E. coli-S. suis shuttle vector; $\mathrm{Spc}^{\mathrm{R}}$ & {$[34]$} \\
\hline pSET2-РcopA-yoeB $B_{V p}$ & $\begin{array}{l}\text { pSET2 containing the yoe } B_{V p} \text { gene and } \\
\text { PcopA promoter }\end{array}$ & This study \\
\hline pSET2-PcopA-yoe $B_{S s}$ & $\begin{array}{l}\text { pSET2 containing the yoe } B_{S s} \text { gene and } \\
\text { PcopA promoter }\end{array}$ & This study \\
\hline
\end{tabular}

\subsection{Preparationof Synthetic Peptide and Natural Transformation Experiment}

The peptide (GNWGTWVEE) was synthesized by Sangon Biotech (Shanghai, China) at $90-95 \%$ purity. It was dissolved in deionized water at a final concentration of $5 \mathrm{mM}$, divided into aliquots of $50 \mu \mathrm{L}$, and stored at $-80^{\circ} \mathrm{C}$.

A natural transformation experiment was performed as previously described [22], with slight modifications. Overnight culture of $S$. suis was diluted 1:100 in fresh medium and grown to an $\mathrm{OD}_{600}$ of $0.035-0.05$ (about 1-2 h). Next, a $100 \mu \mathrm{L}$ sample was removed from the culture; $5 \mu \mathrm{L}$ of the peptide and $1.2 \mu \mathrm{g}$ of DNA (plasmid or PCR products) were added to the sample. Following $2 \mathrm{~h}$ of incubation, the sample was plated on agar plates containing spectinomycin or diluted in fresh media containing $0.5 \mathrm{mM} \mathrm{CuSO}_{4}$.

\subsection{Construction of a S. suis Strain Expressing the Copper-Inducible Yoe $B_{V p}$ Toxin}

The promoter PcopA was amplified from the S. suis SC19 genome using primers PcopA-F/PcopA-R. The DNA fragment containing yoe $B_{V p}$ and its terminator was amplified from $V$. parahaemolyticus RIMD 2210633 genome using primers yoe $B_{V p}-\mathrm{F} /$ yoe $B_{V p}-\mathrm{R}$. The two DNA fragments were fused into a fragment using overlap PCR with primers PcopA-F/yoe $B_{V p}-\mathrm{R}$. Following digestion with the BamH I and EcoR I enzymes, the fused DNA fragment was cloned into the pSET2 vector, to generate pSET2-PcopA-yoe $B_{V p}$. Next, the vector was introduced into the $S$. suis SC19 strain by natural transformation. The resultant strain, SC19/pSET2-PcopA-yoe $B_{V p}$ was confirmed by PCR, DNA sequencing, and reverse transcription quantitative PCR (RT-qPCR).

\subsection{RNA Extraction}

The SC19/pSET2-PcopA-yoe $B_{V p}$ strain was grown to an $\mathrm{OD}_{600}$ of 0.6-0.8 and divided into four aliquots of $1 \mathrm{~mL}$, which were supplemented with deionized water or $\mathrm{CuSO}_{4}$ at final concentrations of $0.1 \mathrm{mM}, 0.2 \mathrm{mM}$, or $0.5 \mathrm{mM}$. After $15 \mathrm{~min}$ of incubation, bacterial cells were collected and subjected to RNA extraction using an Eastep Super Total RNA Isolation Kit (Promega, Shanghai, China). RNA was evaluated for integrity by gel electrophoresis and determined for concentration using a Nanodrop 200.

In another assay, the WT and $\Delta p e r R$ strains were grown to an $\mathrm{OD}_{600}$ of $0.6-0.8$; each strain was then divided into four aliquots of $1 \mathrm{~mL}$. Three of the aliquots were supplemented with $1 \mathrm{mM} \mathrm{FeSO}_{4}, 1 \mathrm{mM} \mathrm{CoSO}_{4}$, and $1 \mathrm{mM} \mathrm{NiSO}_{4}$, respectively; the remaining aliquot was supplemented with deionized water. After $15 \mathrm{~min}$ of incubation, bacterial cells were collected for RNA extraction.

\subsection{RT-qPCR Analysis}

cDNA was generated from approximately $0.2 \mu \mathrm{g}$ of RNA using the NovoScript Plus All-in-one 1st Strand cDNA Synthesis SuperMix (gDNA Purge) (novoprotein, Shanghai, China). Quantitative PCR was performed using NovoStart SYBR qPCR SuperMix Plus (novoprotein, Shanghai, China) and the specific primers listed in Table S1. The reaction mixture was as follows: $2 \times$ NovoStart SYBR qPCR SuperMix Plus $10 \mu \mathrm{L}$, each primer $0.5 \mu \mathrm{M}, 10$-fold diluted cDNA $1 \mu \mathrm{L}$, ROX $0.4 \mu \mathrm{L}$, and finally RNase-free water added to $20 \mu \mathrm{L}$. Quantitative PCR was conducted on the StepOnePlus Real-Time PCR System (Applied Biosystems). The procedure was $95^{\circ} \mathrm{C}$ for $1 \mathrm{~min}$, followed by 40 cycles of $95^{\circ} \mathrm{C}$ 
for $20 \mathrm{~s}$, and $60{ }^{\circ} \mathrm{C}$ for $1 \mathrm{~min}$. A melting curve analysis (starting from $60{ }^{\circ} \mathrm{C}$ and continuing to $95^{\circ} \mathrm{C}$, with $0.3^{\circ} \mathrm{C}$ increments for $5 \mathrm{~s}$ each) was performed to verify the specificity of the products. The amplification efficiency of each primer pair was determined using serially diluted genomic DNA as the template. The gene expression level was calculated using the $2^{-\Delta \Delta C T}$ method [35], with $16 \mathrm{~S}$ rRNA as the reference gene.

\subsection{Growth Curves Analyses}

Overnight cultures of the SC19/pSET2-PcopA-yoeB $B_{V p}$ and SC19/pSET2 strains were diluted in fresh medium and grown to an $\mathrm{OD}_{600}$ of approximately 0.3. Next, each culture was divided into five aliquots ( $1 \mathrm{~mL}$ per aliquot), to which $\mathrm{CuSO}_{4}$ was added at final concentrations of $0,0.05,0.1,0.2$, and $0.5 \mathrm{mM}$, respectively. Each aliquot was sub-packed in triplicate in 96-well plates $(200 \mu \mathrm{L} /$ well $)$ and cultured at $37^{\circ} \mathrm{C}$ for $6 \mathrm{~h}$. The $\mathrm{OD}_{595}$ values were measured hourly using the CMax Plus plate reader (Molecular Devices, Shanghai, China).

\subsection{Spot Dilution Assays}

Overnight cultures of the SC19/pSET2-PcopA-yoe $B_{V p}$ and SC19/pSET2 strains were diluted in fresh medium and grown to an $\mathrm{OD}_{600}$ of approximately 0.6. Next, each culture was serially diluted 10 -fold up to $10^{-5}$ dilution, and $5 \mu \mathrm{l}$ of each dilution was spotted onto the plates supplemented with varying concentrations of $\mathrm{CuSO}_{4}(0,0.1,0.2$, and $0.5 \mathrm{mM})$. The plates were photographically documented following $18 \mathrm{~h}$ of incubation at $37^{\circ} \mathrm{C}$.

\subsection{Construction of the SCIY Positive-Negative Selectable Cassette}

The spectinomycin-resistance gene was amplified from pSET2 using primers $s p c-\mathrm{F} / s p c-\mathrm{R}$. The Pcop $A-y o e B_{V p}$ construct was amplified from pSET2-PcopA-yoe $B_{V p}$ using primers PcopAyoe $B_{V p}-\mathrm{F} /$ Pcop $A-y o e B_{V p}-\mathrm{R}$. The two DNA fragments were fused into a fragment using overlap PCR with primers $s p c-F / P c o p A-y o e B_{V p}-\mathrm{R}$. The fused DNA fragment was confirmed by DNA sequencing, and designated SCIY.

\subsection{Construction of Markerless Gene Deletion Mutants Using the SCIY Cassette}

The $\Delta p m t A$ mutant was constructed using the SCIY cassette via a two-step procedure. For the first step, the left and right arms of pmtA were amplified from $S$. suis SC19 genome using primer pairs $p m t A-\mathrm{LA}-\mathrm{F} / p m t A$-Fir-LA-R and $p m t A-F i r-\mathrm{RA}-\mathrm{F} / p m t A-\mathrm{RA}-\mathrm{R}$, respectively. The SCIY cassette was amplified using primers $p m t A-\mathrm{SCIY}-\mathrm{F} / p m t A-\mathrm{SCIY}-\mathrm{R}$. The three DNA fragments were fused into a fragment using overlap PCR with primers pmtA-LA-F/pmtA-RA-R. The fused DNA fragment was transformed into S. suis SC19 by natural transformation. The spectinomycin-resistant colonies were selected, confirmed by PCR, and designated the intermediate strain. For the second step, the left and right arms of $p m t A$ were amplified from the $S$. suis SC19 genome using primer pairs $p m t A-\mathrm{LA}-\mathrm{F} / p m t A-$ Sec-LA-R and $p m t A-S e c-R A-F / p m t A-R A-R$, respectively. The two DNA fragments were fused into a fragment using overlap PCR with primers $p m t A-\mathrm{LA}-\mathrm{F} / p m t A-\mathrm{RA}-\mathrm{R}$. The fused DNA fragment was transformed into the intermediate strain by natural transformation. Following $2 \mathrm{~h}$ of incubation, the sample was diluted 1:100 in fresh medium containing $0.5 \mathrm{mM} \mathrm{CuSO}_{4}$ and cultured at $37^{\circ} \mathrm{C}$ for another $12 \mathrm{~h}$. In total, the culture was repeatedly diluted three to five times for enrichment of the mutant. After each incubation, the culture was diluted and plated on agar plates. One hundred colonies were tested for spectinomycinsensitivity. Spectinomycin-sensitive colonies were selected, and the absence of $p m t A$ was confirmed by PCR using primer pairs $p m t A$-in-F/pmt $A$-in-R and $p m t A$-out-F/pmt $A$-out-R. The efficiency of the SCIY cassette for counterselection was evaluated as the proportion of spectinomycin-sensitive colonies. The $\Delta p e r R$ and $\Delta l y s R$ mutants were constructed using the same procedure to verify the method. 


\section{Results}

3.1. Identification of the S. suis Strain Expressing the Copper-Inducible YoeB ${ }_{V p}$ Toxin

To evaluate the effect of $Y_{0 e B_{V p}}$ induction on $S$. suis growth, we constructed a S. suis strain expressing the copper-inducible $\mathrm{YoeB}_{V p}$ toxin using the PcopA promoter and pSET2 vector $[34,36]$. The strain, termed SC19/pSET2-PcopA-yoeB $B_{V p}$, was identified by PCR (Figure 1A) and DNA sequencing (data not shown). RT-qPCR analysis was also performed to detect whether copper can induce yoe $B_{V p}$ expression. As shown in Figure 1B, the expression of yoe $B_{V p}$ was significantly induced by copper, and the inductive effects increased with increasing copper concentrations.
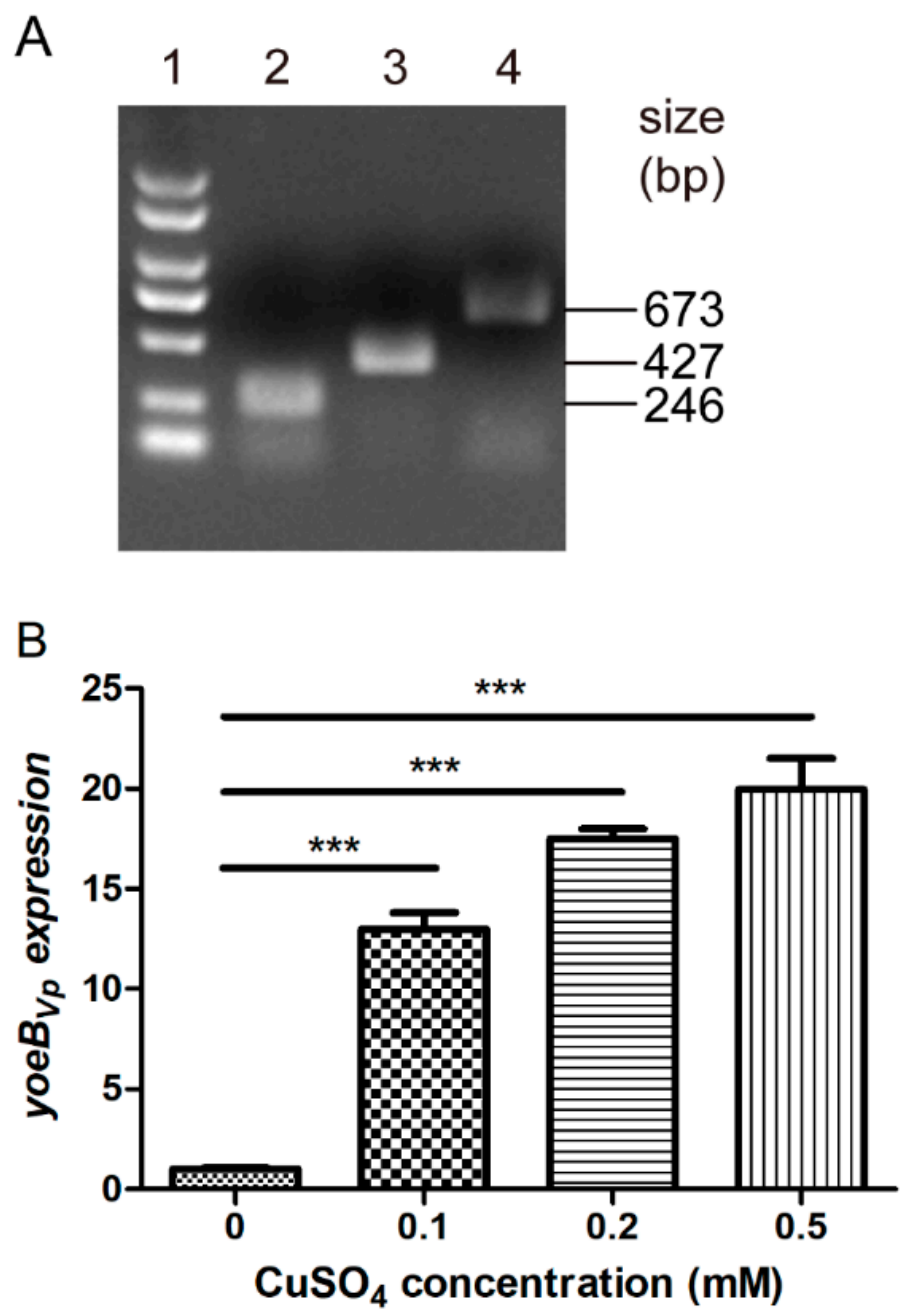

Figure 1. Identification of the $S$. suis strain expressing the copper-inducible $Y_{0 e B_{V p}}$ toxin. (A) PCR identification of the SC19/pSET2-PcopA-yoe $B_{V p}$ strain. Lane 1 indicates the DL2000 DNA marker. Lanes 2-4 indicate PCR amplification of the PcopA promoter, the yoe $B_{V p}$ gene, and the PcopA-yoe $B_{V p}$ construct, respectively. (B) RT-qPCR identification of the SC19/pSET2-PcopA-yoe $B_{V p}$ strain. The data shown are the means and standard deviations (SD) from three independent experiments. One-way analysis of variance with Bonferroni's post-test was used for statistical analyses. ${ }^{* * *}$ indicates $p<0.001$.

\subsection{YoeBVp Expression Results in Growth Defect in S. suis}

The SC19/pSET2-PcopA-yoeB $V p$ and SC19/pSET2 strains were grown in fresh media containing various concentrations of $\mathrm{CuSO}_{4}$, and their growth curves were measured. As shown in Figure 2A, the two strains exhibited similar growth in the absence of $\mathrm{CuSO}_{4}$. However, when supplemented with $\mathrm{CuSO}_{4}$, the SC19/pSET2-PcopA-yoeB $B_{V p}$ strain displayed a remarkable growth defect compared with the SC19/pSET2 strain (Figure 2B-D). 

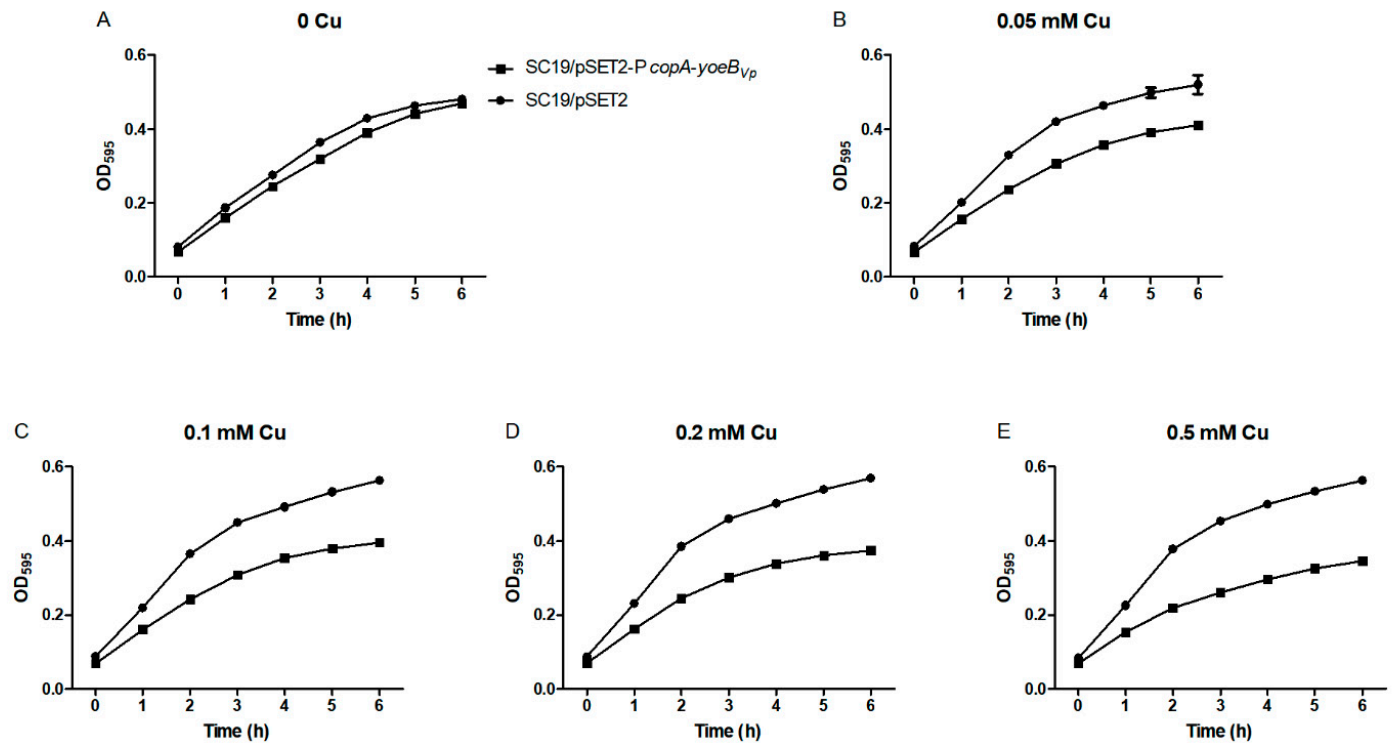

Figure 2. YoeB $B_{V p}$ expression resulted in a growth defect in S. suis in liquid media. The SC19/pSET2-PcopA-yoeB $V p$ and SC19/pSET2 strains were grown in the absence $(\mathbf{A})$ and presence of various concentrations of $\mathrm{CuSO}_{4}(\mathbf{B}-\mathbf{E}) ;(\mathbf{B}) 0.05 \mathrm{mM}$ $\mathrm{CuSO}_{4}$; (C) $0.1 \mathrm{mM} \mathrm{CuSO}_{4}$; (D) $0.2 \mathrm{mM} \mathrm{CuSO}_{4}$; (E) $0.5 \mathrm{mM} \mathrm{CuSO}_{4}$. At least three independent experiments were performed; the data shown are the means \pm SDs from three wells in a representative experiment.

The effect of $Y_{0 e B_{V p}}$ expression on $S$. suis growth was also detected on agar plates. In the absence of $\mathrm{CuSO}_{4}$, the two strains formed colonies of equal sizes (Figure 3). However, in the presence of $\mathrm{CuSO}_{4}$, the SC19/pSET2-PcopA-yoeB $V p$ strain formed colonies of smaller sizes than the SC19/pSET2 strain (Figure 3).

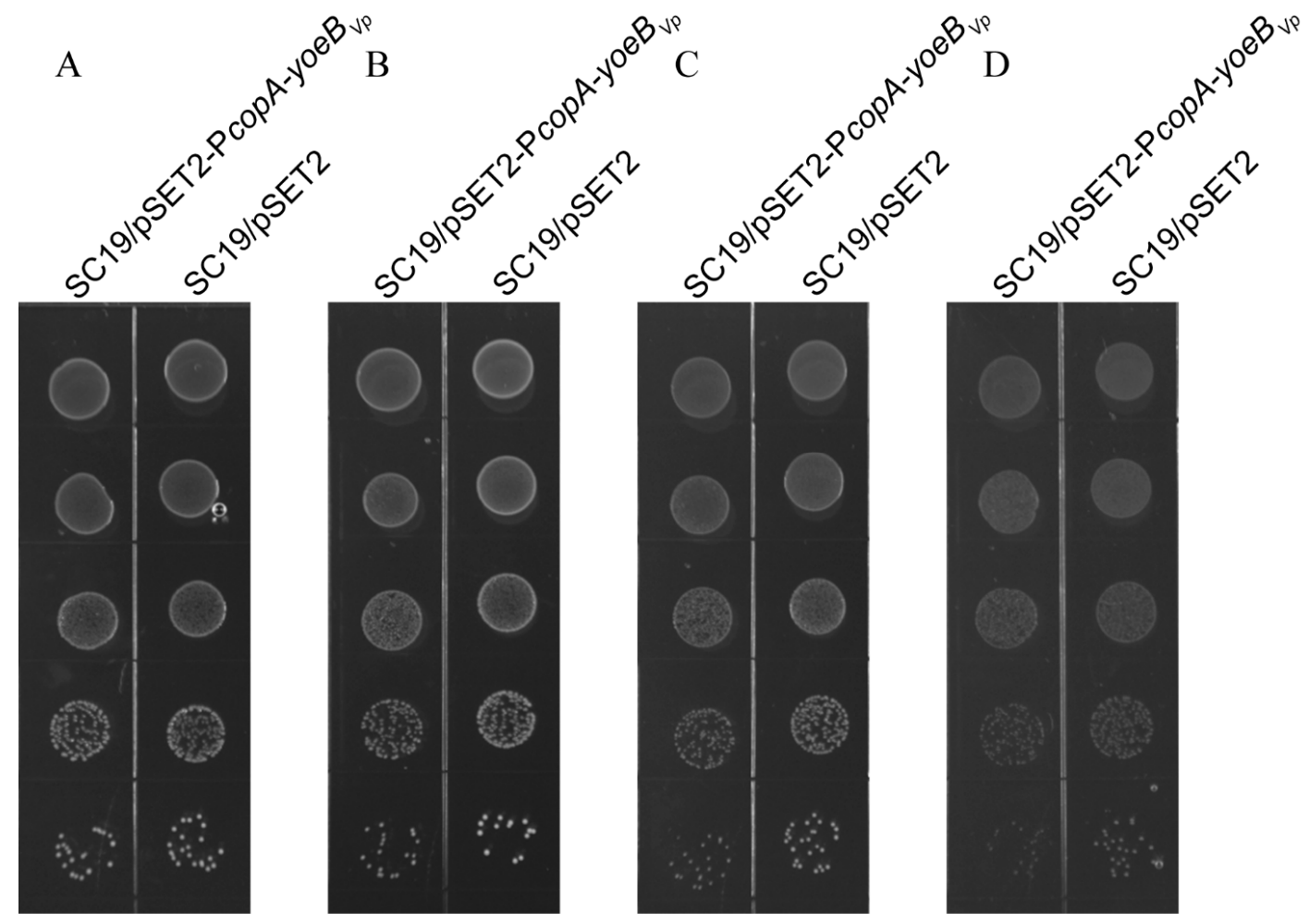

Figure 3. YoeB ${ }_{V p}$ expression resulted in growth defect in S. suis on agar plates. The SC19/pSET2-PcopA-yoeB $B_{V p}$ and SC19/pSET2 strains were grown in the absence (A) and presence of various concentrations of CuSO 4 (B-D); (B) $0.1 \mathrm{mM}$ $\mathrm{CuSO}_{4}$; (C) $0.2 \mathrm{mM} \mathrm{CuSO}_{4}$; (D) $0.5 \mathrm{mM} \mathrm{CuSO}_{4}$. The images are representative of at least three independent experiments. 
Taken together, $Y_{0 e} B_{V p}$ expression in S. suis led to growth inhibition both in liquid media and on agar plates. Thus, $Y_{o e} B_{\mathrm{Vp}}$ has the potential to be a counterselectable marker for S. suis.

\subsection{Establishment of the Cloning-Independent and Counterselectable Markerless Gene Deletion System in S. suis}

The spectinomycin-resistance gene and PcopA-yoe $B_{V p}$ construct were combined to generate the SCIY cassette, which was further used for markerless gene deletion in S. suis. The strategy for markerless gene deletion in S. suis using the SCIY cassette is shown in Figure 4. In the first step, an intermediate strain was generated, in which the SCIY cassette replaced the target gene. As the SCIY cassette contains the spectinomycin-resistance gene, the intermediate strain could be selected with spectinomycin. In the second step, the markerless gene deletion mutant was generated. The intermediate strain contains the PcopA-yoeB $V p$ construct; thus, its growth was inhibited in the presence of copper. However, the mutant could grow well in the presence of copper. After three to five dilutions in media supplemented with copper, the mutant was enriched to be easily isolated.

Step 1

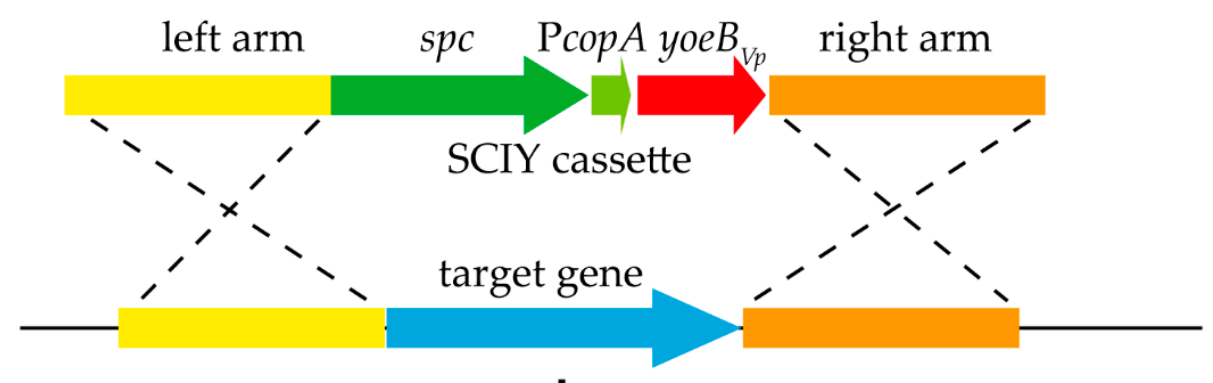

Wild type

Selection with spectinomycin

Intermediate strain

Step 2

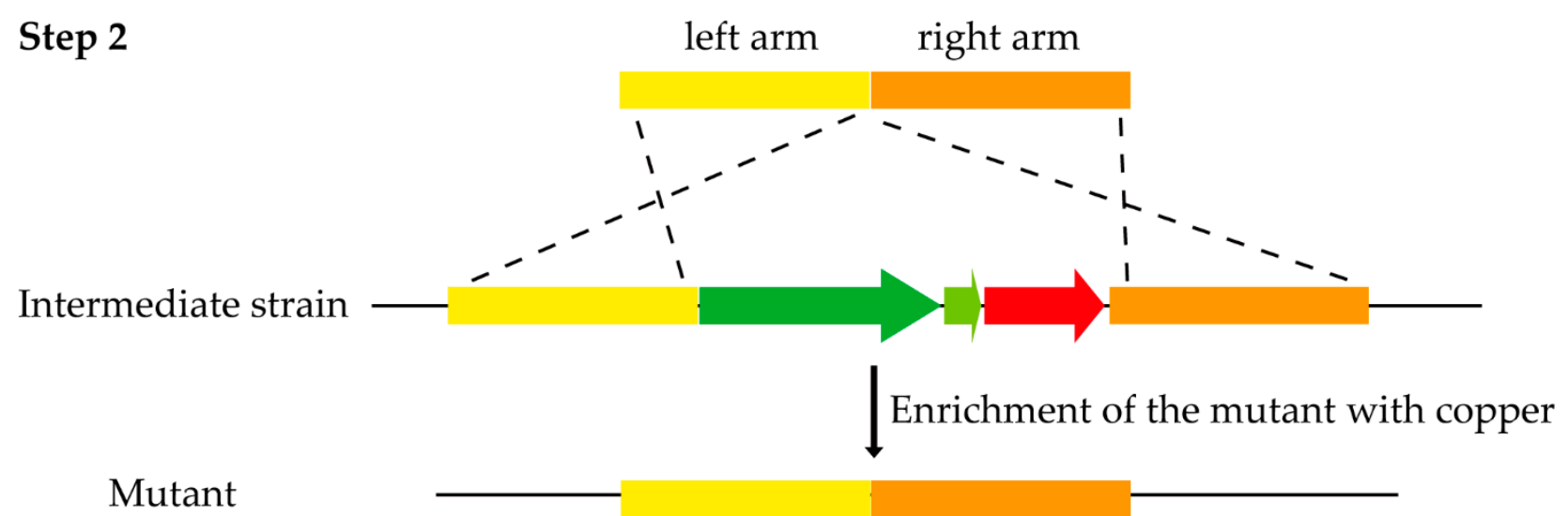

Intermediate strain

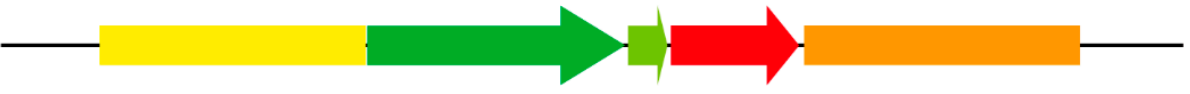

Figure 4. Schematic representation of the two-step markerless gene deletion method in S. suis. In the first step, the target gene was replaced by the SCIY cassette. The intermediate strain was selected with spectinomycin. In the second step, the markerless gene deletion mutant was generated. Growth of the intermediate strain was inhibited by copper, whereas the mutant was tolerant to copper. The medium was supplemented with copper for the enrichment of the mutant.

\subsection{Markerless Deletion of the pmtA, perR, and lysR Genes in S. suis}

To assess whether the strategy is effective, we firstly constructed a markerless deletion mutant of the $p m t A$ gene. As seen in Figure 5A, PCR amplification of the $\triangle p m t A$ mutant using primers $p m t A$-in-F/pmt $A$-in-R generated no products, whereas amplification of the WT 
strain generated products with expected sizes (755 bp). Furthermore, PCR amplification of $\triangle p m t A$ and the WT strain using primers $p m t A$-out-F/pmtA-out-R generated products with expected sizes for $\triangle p m t A$ (2472 bp) and the WT strain (4199 bp), respectively (Figure 5A). DNA sequencing confirmed that the $p m t A$ gene was successfully deleted in the $\Delta p m t A$ mutant. To further verify the strategy, markerless deletion mutants of the perR (Figure $5 \mathrm{~B}$ ) and $l y s R$ genes (Figure 5C) were also constructed. Overall, the two-step strategy applying the SCIY cassette is effective in markerless gene deletion in S. suis.

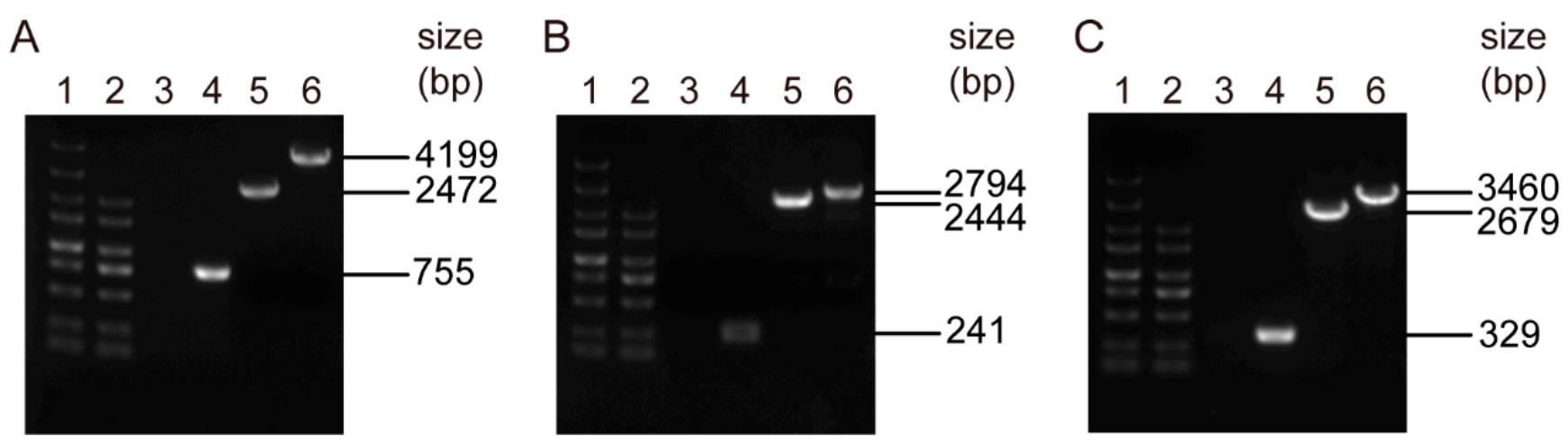

Figure 5. PCR identification of the mutants. (A) $\Delta p m t A$; (B) $\Delta p e r R ;$ (C) $\Delta l y s R$. Lanes 1 and 2 indicate the DL5000 and DL2000 DNA markers, respectively. Lanes 3 and 5 indicate PCR amplification of the mutants using the primer pairs in-F/in-R and out-F/out-R, respectively. Lanes 4 and 6 indicate PCR amplification of the WT strain using the primer pairs in-F/in-R and out-F/out-R, respectively.

\subsection{The SCIY Cassette Is Highly Efficient for Counterselection in S. suis}

The proportion of the mutant after each subculture was evaluated to determine SCIY counterselection efficiency in S. suis. As shown in Table 2, approximately $95 \%$ of the colonies were the $\triangle p m t A$ mutant after subculturing three times. For the perR and lysR genes, approximately half or greater than $80 \%$ of the colonies were the mutant strain after five times of subculture (Table 2). The results indicate that the SCIY cassette is highly efficient as a counterselectable marker for S. suis.

Table 2. The proportion of spectinomycin-sensitive colonies (mutants).

\begin{tabular}{ccccccc}
\hline Gene & Repetition & \multicolumn{5}{c}{ Spectinomycin-Sensitive Colonies (Mutants) (\%) } \\
\hline \multirow{6}{*}{ pmtA } & $\begin{array}{c}\text { First } \\
\text { Dilution }\end{array}$ & $\begin{array}{c}\text { Second } \\
\text { Dilution }\end{array}$ & $\begin{array}{c}\text { Third } \\
\text { Dilution }\end{array}$ & $\begin{array}{c}\text { Fourth } \\
\text { Dilution }\end{array}$ & $\begin{array}{c}\text { Fifth } \\
\text { Dilution }\end{array}$ \\
\hline \multirow{6}{*}{ perR } & Rep_1 & 5 & 75 & 98 & & \\
& Rep_2 & 3 & 91 & 98 & & \\
& Rep_3 & 1 & 65 & 95 & & \\
& Rep_1 & 0 & 0 & 63 & 57 & 49 \\
& Rep_2 & 0 & 32 & 98 & 100 & 98 \\
& Rep_3 & 0 & 5 & 83 & 93 & 97 \\
& Rep_1 & 2 & 1 & 27 & 24 & 86 \\
& Rep_2 & 0 & 1 & 0 & 15 & 52 \\
& Rep_3 & 2 & 0 & 1 & 32 & 87 \\
\hline
\end{tabular}

${ }^{1}$ The percentage of spectinomycin-sensitive colonies (mutants) was determined by analysis of 100 colonies. The experiments were performed three times for deletion of each gene, and the results for each repetition are shown.

\subsection{PerR Is a Transcriptional Repressor of the Ferrous Iron and Cobalt Efflux Pump in S. suis}

In a previous study, we demonstrated that the $p m t A$ gene encodes a ferrous iron and cobalt efflux pump in S. suis; its expression was significantly induced by ferrous iron, cobalt, and nickel [32]. Upstream of the $p m t A$ gene is a gene encoding the PerR regulator. RT-qPCR analysis was performed to determine whether the $p m t A$ gene is under the control of PerR. As shown in Figure 6, pmtA expression in the WT strain was upregulated following 
treatment with ferrous iron, cobalt, and nickel. However, pmt $A$ expression in the $\Delta p e r R$ mutant was upregulated without metal supplementation (Figure 6). The results reveal that deletion of perR led to derepression of the $p m t A$ gene; thus, $p m t A$ expression in $\Delta p e r R$ was upregulated without treatment with ferrous iron, cobalt, or nickel.

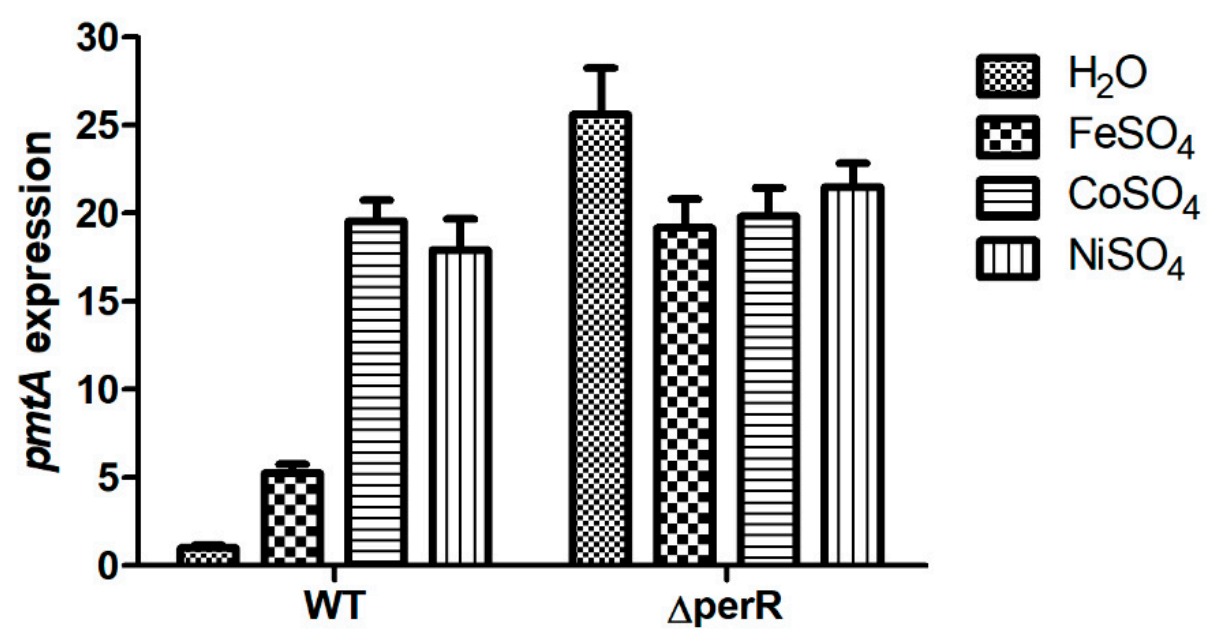

Figure 6. pmt $A$ expression in the WT and $\Delta$ perR strains in the absence and presence of ferrous iron, cobalt, and nickel. The data shown are the means and standard deviations (SD) from three independent experiments.

\section{Discussion}

S. suis is an important zoonotic pathogen that causes severe infections in swine and humans. Research on the physiology and pathogenesis of $S$. suis usually relies on gene deletion mutants. In the present study, we describe a novel two-step method for markerless gene deletion in S. suis. This method is established based on natural transformation in S. suis [22] and the utilization of V. parahaemolyticus YoeB toxin as a counterselectable marker.

TA systems are widely prevalent in bacteria and archaea [23]. Some toxin genes have been developed as counterselectable markers for genetic manipulation based on toxins' antibacterial activity [26-30]. In a previous study, induction of $V$. parahaemolyticus YoeB toxin in E. coli was found to cause cell death [31]. This finding led to the speculation that $Y_{o e} B_{V p}$ could be an ideal counterselectable marker. YoeB $V p$ expression should be precisely controlled to be an available counterselectable marker. In S. suis, the copA gene, which encodes a copper efflux system, could be specifically induced by copper [36]. The promoter PcopA might be reliable to control $\mathrm{YoeB}_{V p}$ expression in $S$. suis. Herein, a $S$. suis strain expressing the copper-inducible $\mathrm{Yoe}_{V p}$ toxin was constructed to test the counterselection potential of $\mathrm{YoeB}_{V p}$. As expected, the addition of copper to the culture induced yoe $B_{V p}$ expression and inhibited $S$. suis growth. It should be noted that a homologous TA system of YefM-YoeB is present in S. suis [37]. We also evaluated the counterselection potential of $Y_{o e B}$. Induction of $Y_{0 e B}$ resulted in drastic growth inhibition in E. coli [37], whereas no growth defect was observed when $Y_{\text {oeB }}$ s was induced in S. suis (Figure S1). We speculate that the endogenous YefM $_{S s}$ antitoxin counteracted the toxicity of YoeB $\mathrm{B}_{S s}$. While YoeB $V p$ shares $63 \%$ identity with YoeB $_{S s}$ at the amino acid level, YefM $V p$ shares only $29 \%$ identity with YefM $_{S s}$. Therefore, it is not surprising that the toxicity of YoeB ${ }_{V p}$ was not counteracted by YefM $S_{S}$.

A SCIY cassette composed of the spectinomycin-resistance gene and PcopA-yoe $B_{V p}$ construct was generated to explore its application for markerless gene deletion in S. suis. The first step, by which the SCIY cassette replaced the target gene, was adopted from a previously described method [22]. The intermediate strain was easily selected from plates containing spectinomycin. Since $\mathrm{YoeB}_{V p}$ toxin exerts a bacteriostatic effect rather than a bactericidal effect on $S$. suis, the mutant generated from the second step should not be selected directly from plates containing copper. Instead, several dilutions in media 
containing copper were performed for the enrichment of the mutant. Our results showed that after subculturing three to five times, the mutant was easy to isolate. However, the mutant's proportion after each subculture should be correlated with the efficiency of natural transformation and homologous recombination.

In a previous study, a cassette containing a kanamycin resistance gene and a gene encoding the ParE toxin has been used to introduce a single mutation in Salmonella Typhimurium [30]. Similarly, the SCIY cassette could be applied in site-directed mutagenesis or deletion of a few bases in the genome of $S$. suis, which is an outstanding advantage of the two-step method. The conventional method using pSET4s generates the mutant and WT genotype simultaneously, which were preliminarily identified by PCR. It would be difficult to distinguish the mutant and WT strains by PCR when only a few bases were deleted. If using the two-step method, the SCIY cassette in the intermediate strain could be easily replaced by the target gene with desired site-directed mutagenesis or a few bases deletion in the second step. The two-step method would facilitate research of the role of a single amino acid or protein domain in S. suis.

Although the two-step method is highly efficient in markerless gene deletion in S. suis, it does not mean that it could not be further improved. Next, the effect of other toxins on $S$. suis growth will be evaluated. If a toxin is found to exert bactericidal activity against $S$. suis, the yoe $B_{V p}$ gene in the SCIY cassette will be replaced by this gene. Then, the intermediate strain is expected to be killed in the presence of copper, so that the mutant can be easily isolated in the second step without enrichment. In addition, some undesired mutations might be introduced into the genome during construction of the mutant. Therefore, it would be better to generate a complementation strain for the mutant when performing a functional study of a gene.

BlastP analysis also revealed that $\mathrm{YefM}_{V p}$ and $\mathrm{YoeB}_{V p}$ share $30 \%$ and $63 \%$ amino acid sequence identity with the homologous antitoxin and toxin from Streptococcus pneumoniae, respectively [38]. It is likely that the $\mathrm{YoeB}_{V p}$ toxin could exert a toxic effect against S. pneumoniae, which might not be counteracted by the endogenous $Y_{\text {efM }} \mathrm{M}_{S p}$ antitoxin. Therefore, further studies could be performed to detect the counterselection potential of the YoeB $_{V p}$ toxin in other species such as $S$. pneumoniae. Yet, a suitable promoter should be selected to control yoe $B_{V p}$ expression in the corresponding species.

In a previous study, we demonstrated that the pmt $A$ gene is involved in ferrous iron and cobalt efflux in $S$. suis [32]. One of the remaining questions is which regulator modulates pmt $A$ expression. In Streptococcus pyogenes, the PmtA homolog is regulated by PerR $[39,40]$. In $S$. suis, the perR gene is located upstream of the $p m t A$ gene. Using the $\Delta p e r R$ mutant generated by the novel two-step method, we demonstrated that in the absence of metal supplementation, $p m t A$ expression in the $\Delta$ perR mutant was significantly upregulated compared to that in the WT strain. This result is consistent with the observations in S. pyogenes $[39,40]$. Thus, PerR is a transcriptional repressor of $p m t A$ in S. suis.

In conclusion, a novel two-step markerless gene deletion method was established for S. suis. This method is cloning-independent and can also be used for site-directed mutagenesis or deletion of a few bases in the genome of $S$. suis. Moreover, we demonstrate that PerR is a transcriptional repressor of ferrous iron and cobalt efflux pump (PmtA) in S. suis.

Supplementary Materials: The following are available online at https:/ /www.mdpi.com/article/10.3 390/microorganisms9051095/s1, Table S1: Primers used in this study. Figure S1: YoeB $B_{S s}$ expression had no significant effect on $S$. suis growth in liquid media. Text S1: DNA sequence of the SCIY cassette.

Author Contributions: Conceptualization, C.Z.; methodology, M.W. and J.Q.; validation, C.Z., M.W. and J.Q.; formal analysis, C.Z.; investigation, M.W.; resources, C.Z.; data curation, J.Q.; writingoriginal draft preparation, C.Z.; writing-review and editing, M.W. and J.L.; visualization, M.W.; supervision, J.L.; project administration, C.Z.; funding acquisition, C.Z. All authors have read and agreed to the published version of the manuscript. 
Funding: This research was funded by the National Natural Science Foundation of China, grant number 31802210; China Postdoctoral Science Foundation, grant number 2018M630615; and the Interdisciplinary Project from Veterinary Science of Yangzhou University, grant number yzuxk202002.

Institutional Review Board Statement: Not applicable.

Informed Consent Statement: Not applicable.

Data Availability Statement: Not applicable.

Acknowledgments: We thank Tsutomu Sekizaki (National Institute of Animal Health, Japan) for supplying the pSET2 vector.

Conflicts of Interest: The authors declare no conflict of interest. The funders had no role in the design of the study; in the collection, analyses, or interpretation of data; in the writing of the manuscript, or in the decision to publish the results.

\section{References}

1. Feng, Y.J.; Zhang, H.M.; Wu, Z.W.; Wang, S.H.; Cao, M.; Hu, D.; Wang, C.J. Streptococcus suis infection An emerging/reemerging challenge of bacterial infectious diseases? Virulence 2014, 5, 477-497. [CrossRef] [PubMed]

2. $\quad$ Lun, Z.R.; Wang, Q.P.; Chen, X.G.; Li, A.X.; Zhu, X.Q. Streptococcus suis: An emerging zoonotic pathogen. Lancet Infect. Dis. 2007, 7, 201-209. [CrossRef]

3. Segura, M.; Aragon, V.; Brockmeier, S.L.; Gebhart, C.; Greeff, A.; Kerdsin, A.; O’Dea, M.A.; Okura, M.; Salery, M.; Schultsz, C.; et al. Update on Streptococcus suis research and prevention in the era of antimicrobial restriction: 4th International Workshop on S. suis. Pathogens 2020, 9, 374. [CrossRef] [PubMed]

4. Zhang, B.; Ku, X.; Yu, X.; Sun, Q.; Wu, H.; Chen, F.; Zhang, X.; Guo, L.; Tang, X.; He, Q. Prevalence and antimicrobial susceptibilities of bacterial pathogens in Chinese pig farms from 2013 to 2017. Sci. Rep. 2019, 9, 9908. [CrossRef]

5. Segura, M.; Calzas, C.; Grenier, D.; Gottschalk, M. Initial steps of the pathogenesis of the infection caused by Streptococcus suis: Fighting against nonspecific defenses. FEBS Lett. 2016, 590, 3772-3799. [CrossRef] [PubMed]

6. Goyette-Desjardins, G.; Auger, J.P.; Xu, J.; Segura, M.; Gottschalk, M. Streptococcus suis, an important pig pathogen and emerging zoonotic agent-an update on the worldwide distribution based on serotyping and sequence typing. Emerg. Microbes Infect. 2014, 3, e45. [CrossRef]

7. Tang, J.; Wang, C.; Feng, Y.; Yang, W.; Song, H.; Chen, Z.; Yu, H.; Pan, X.; Zhou, X.; Wang, H.; et al. Streptococcal toxic shock syndrome caused by Streptococcus suis serotype. PLoS Med. 2006, 3, e151.

8. Yu, H.; Jing, H.; Chen, Z.; Zheng, H.; Zhu, X.; Wang, H.; Wang, S.; Liu, L.; Zu, R.; Luo, L.; et al. Human Streptococcus suis outbreak, Sichuan, China. Emerg. Infect. Dis. 2006, 12, 914-920. [CrossRef]

9. Romay-Lema, E.M.; Ventura-Valcárcel, P.; Iñiguez-Vázquez, I.; García-Pais, M.J.; García-Garrote, F.; Rabuñal-Rey, R.; Alonso, M.P.; Corredoira-Sánchez, J. Streptococcus suis spondylodiscitis: 2 new cases and a literature review. Enferm. Infecc. Microbiol. Clin. 2020. [CrossRef]

10. Agoston, Z.; Terhes, G.; Hannauer, P.; Gajdacs, M.; Urban, E. Fatal case of bacteremia caused by Streptococcus suis in a splenectomized man and a review of the European literature. Acta Microbiol. Immunol. Hung. 2020, 67, 148-155. [CrossRef]

11. Jiang, F.; Guo, J.; Cheng, C.; Gu, B. Human infection caused by Streptococcus suis serotype 2 in China: Report of two cases and epidemic distribution based on sequence type. BMC Infect. Dis. 2020, 20, 223. [CrossRef]

12. Olearo, F.; Marinosci, A.; Stephan, R.; Cherkaoui, A.; Renzi, G.; Gaia, N.; Leo, S.; Lazarevic, V.; Schrenzel, J. First case of Streptococcus suis infection in Switzerland: An emerging public health problem? Travel Med. Infect. Dis. 2020, $36,101590$. [CrossRef]

13. Liu, S.S.; Wang, Y.; Xue, L.; Ma, C.; Li, C.H. Hemophagocytic lymphohistiocytosis due to Streptococcus suis in a 12-year-old girl: A case report. Medicine 2019, 98, e15136. [CrossRef]

14. Németh, A.; Knausz, M.; Schmidt, P. Special case of purulent meningitis caused by Streptococcus suis. Case report. Orv. Hetil. 2019, 160, 30-34. [CrossRef]

15. Segura, M.; Fittipaldi, N.; Calzas, C.; Gottschalk, M. Critical Streptococcus suis virulence factors: Are they all really critical? Trends Microbiol. 2017, 25, 586-594. [CrossRef]

16. Fittipaldi, N.; Segura, M.; Grenier, D.; Gottschalk, M. Virulence factors involved in the pathogenesis of the infection caused by the swine pathogen and zoonotic agent Streptococcus suis. Future Microbiol. 2012, 7, 259-279. [CrossRef]

17. Arenas, J.; Bossers-de Vries, R.; Harders-Westerveen, J.; Buys, H.; Ruuls-van Stalle, L.M.F.; Stockhofe-Zurwieden, N.; Zaccaria, E.; Tommassen, J.; Wells, J.M.; Smith, H.E.; et al. In vivo transcriptomes of Streptococcus suis reveal genes required for niche-specific adaptation and pathogenesis. Virulence 2019, 10, 334-351. [CrossRef]

18. Arenas, J.; Zomer, A.; Harders-Westerveen, J.; Bootsma, H.J.; Jonge, M.I.D.; Stockhofe-Zurwieden, N.; Smith, H.E.; Greeff, A.D. Identification of conditionally essential genes for Streptococcus suis infection in pigs. Virulence 2020, 11, 446-464. [CrossRef]

19. Pei, X.; Liu, M.; Zhou, H.; Fan, H. Screening for phagocytosis resistance-related genes via a transposon mutant library of Streptococcus suis serotype. Virulence 2020, 11, 825-838. [CrossRef] 
20. Takamatsu, D.; Osaki, M.; Sekizaki, T. Thermosensitive suicide vectors for gene replacement in Streptococcus suis. Plasmid 2001, 46, 140-148. [CrossRef]

21. Zhu, Y.; Dong, W.; Ma, J.; Zhang, Y.; Pan, Z.; Yao, H. Utilization of the ComRS system for the rapid markerless deletion of chromosomal genes in Streptococcus suis. Future Microbiol. 2019, 14, 207-222. [CrossRef] [PubMed]

22. Zaccaria, E.; Van Baarlen, P.; Greeff, A.D.; Morrison, D.A.; Smith, H.; Wells, J.M. Control of competence for DNA transformation in Streptococcus suis by genetically transferable pherotypes. PLoS ONE 2014, 9, e99394. [CrossRef]

23. Unterholzner, S.J.; Poppenberger, B.; Rozhon, W. Toxin-antitoxin systems: Biology, identification, and application. Mob. Genet. Elements 2013, 3, e26219. [CrossRef] [PubMed]

24. Díaz-Orejas, R.; Espinosa, M.; Yeo, C.C. The importance of the expendable: Toxin-antitoxin genes in plasmids and chromosomes. Front Microbiol. 2017, 8, 1479. [CrossRef] [PubMed]

25. Harms, A.; Brodersen, D.E.; Mitarai, N.; Gerdes, K. Toxins, targets, and triggers: An overview of toxin-antitoxin biology. Mol. Cell 2018, 70, 768-784. [CrossRef]

26. Song, P.P.; Liu, S.; Guo, X.N.; Bai, X.J.; He, X.P.; Zhang, B.R. Scarless gene deletion in methylotrophic Hansenula polymorpha by using mazF as counter-selectable marker. Anal. Biochem. 2015, 468, 66-74. [CrossRef] [PubMed]

27. Zhang, X.Z.; Yan, X.; Cui, Z.L.; Hong, Q.; Li, S.P. mazF, a novel counter-selectable marker for unmarked chromosomal manipulation in Bacillus subtilis. Nucleic Acids Res. 2006, 34, e71. [CrossRef]

28. Luo, P.; He, X.; Liu, Q.; Hu, C. Developing universal genetic tools for rapid and efficient deletion mutation in Vibrio species based on suicide T-vectors carrying a novel counterselectable marker, vmi. PLoS ONE 2015, 10, e0144465. [CrossRef]

29. Song, Q.; Li, Z.; Chen, R.; Ma, X.; Xiao, X.; Xu, J. Induction of a toxin-antitoxin gene cassette under high hydrostatic pressure enables markerless gene disruption in the hyperthermophilic archaeon Pyrococcus yayanosii. Appl. Environ. Microbiol. 2019, 85, e02662-18. [CrossRef]

30. Lobato-Márquez, D.; Molina-García, L.; Moreno-Córdoba, I.; García-Del Portillo, F.; Díaz-Orejas, R. Stabilization of the virulence plasmid pSLT of Salmonella Typhimurium by three maintenance systems and its evaluation by using a new stability test. Front. Mol. Biosci. 2016, 3, 66. [CrossRef]

31. Wei, M.; Qiu, J.; Jia, M.; Zheng, C. Identification of the chromosomal YefM-YoeB toxin-antitoxin system in Vibrio parahemolyticus. Microbiol. China 2021, 1-14. Available online: http:/ / cn.oversea.cnki.net/KCMS / detail/detail.aspx?filename=WSWT202102020 00\&dbcode=CJFQ\&dbname=DKFX2021 (accessed on 1 April 2021).

32. Zheng, C.; Jia, M.; Gao, M.; Lu, T.; Li, L.; Zhou, P. PmtA functions as a ferrous iron and cobalt efflux pump in Streptococcus suis. Emerg. Microbes Infect. 2019, 8, 1254-1264. [CrossRef]

33. Teng, L.; Dong, X.; Zhou, Y.; Li, Z.; Deng, L.; Chen, H.; Wang, X.; Li, J. Draft genome sequence of hypervirulent and vaccine candidate Streptococcus suis strain SC19. Genome Announc. 2017, 5, e01484-16. [CrossRef]

34. Takamatsu, D.; Osaki, M.; Sekizaki, T. Construction and characterization of Streptococcus suis-Escherichia coli shuttle cloning vectors. Plasmid 2001, 45, 101-113. [CrossRef]

35. Livak, K.J.; Schmittgen, T.D. Analysis of relative gene expression data using real-time quantitative PCR and the $2^{-\Delta \Delta C_{\mathrm{T}}}$ method. Methods 2001, 25, 402-408. [CrossRef] [PubMed]

36. Zheng, C.; Jia, M.; Lu, T.; Gao, M.; Li, L. CopA protects Streptococcus suis against copper toxicity. Int. J. Mol. Sci. 2019, 20, 2969. [CrossRef]

37. Zheng, C.; Xu, J.; Ren, S.; Li, J.; Xia, M.; Chen, H.; Bei, W. Identification and characterization of the chromosomal yefM-yoeB toxin-antitoxin system of Streptococcus suis. Sci. Rep. 2015, 5, 13125. [CrossRef]

38. Chan, W.T.; Nieto, C.; Harikrishna, J.A.; Khoo, S.K.; Othman, R.Y.; Espinosa, M.; Yeo, C.C. Genetic regulation of the yefM-yoeB toxin-antitoxin locus of Streptococcus pneumoniae. J. Bacteriol. 2011, 193, 4612-4625. [CrossRef]

39. Turner, A.G.; Ong, C.L.Y.; Djoko, K.Y.; West, N.P.; Davies, M.R.; McEwan, A.G.; Walker, M.J. The PerR-regulated P1B-4-type ATPase (PmtA) acts as a ferrous iron efflux pump in Streptococcus pyogenes. Infect. Immun. 2017, 85, e00140-17. [CrossRef]

40. Brenot, A.; Weston, B.F.; Caparon, M.G. A PerR-regulated metal transporter (PmtA) is an interface between oxidative stress and metal homeostasis in Streptococcus pyogenes. Mol. Microbiol. 2007, 63, 1185-1196. [CrossRef] [PubMed] 